\title{
A note on testing the covariance matrix for large dimension
}

\author{
Melanie Birke \\ Ruhr-Universität Bochum \\ Fakultät für Mathematik \\ 44780 Bochum, Germany \\ e-mail: melanie.birke@ruhr-uni-bochum.de
}

\author{
Holger Dette \\ Ruhr-Universität Bochum \\ Fakultät für Mathematik \\ 44780 Bochum, Germany \\ e-mail: holger.dette@ruhr-uni-bochum.de
}

FAX: +492343214559

December 19, 2003

\begin{abstract}
We consider the problem of testing hypotheses regarding the covariance matrix of multivariate normal data, if the sample size $s$ and dimension $n$ satisfy $\lim _{n, s \rightarrow \infty} n / s=y$. Recently, several tests have been proposed in the case, where the sample size and dimension are of the same order, that is $y \in(0, \infty)$. In this paper we consider the cases $y=0$ and $y=\infty$. It is demonstrated that standard techniques are not applicable to deal with these cases. A new technique is introduced, which is of its own interest, and is used to derive the asymptotic distribution of the test statistics in the extreme cases $y=0$ and $y=\infty$.
\end{abstract}

AMS Subject Classification 62H15; 62E20

Keywords and phrases: sphericity test, random matrices, Wishart distribution

\section{Introduction}

A common assumption in multivariate statistics is that of an i.i.d. sample of normally distributed $n$-dimensional random variables $X_{1}, \ldots, X_{s+1} \sim \mathcal{N}(\mu, \Sigma)$, where $\mu \in \mathbb{R}^{n}$ denotes the mean vector and $\Sigma \in \mathbb{R}^{n \times n}$ a positive definite covariance matrix. In this paper we are interested in the problems of testing the hypothesis of sphericity, that is

$$
H_{0}: \exists \sigma \in \mathbb{R}^{>0}: \Sigma=\sigma I_{n}, \quad H_{1}: \Sigma \neq \sigma I_{n} \forall \sigma \in \mathbb{R}^{>0},
$$

and in the problem of testing the hypothesis

$$
H_{0}^{\prime}: \Sigma=I_{n}, \quad H_{1}^{\prime}: \Sigma \neq I_{n}
$$


if the sample size $s$ and dimension $n$ converge to infinity, where $I_{n} \in \mathbb{R}^{n \times n}$ denotes the identity matrix. Note that the identity matrix $I_{n}$ in (1.2) can be replaced by any other positive definite matrix $\Sigma_{0} \in \mathbb{R}^{n \times n}$ by multiplying the data by $\Sigma_{0}^{-1 / 2}$. Classical analysis assumes that the dimension is fixed, while the sample size converges to infinity. However, recent applications in gene express experiments require statistical methods, which are applicable in the case, where the dimension is large compared to the sample size [see Sebastini, Gussoni, Kohane and Ramoni (2003)]. Because likelihood ratio tests [see e.g. Muirhead (1982) or Anderson (1984)] are not applicable in the case $n \geq s$, Ledoit and Wolf (2002) suggested to use certain alternative tests which were originally proposed by John (1971) and Nagao (1973) in the case, where the dimension $n$ is fixed and the sample size converges to infinity. To be precise, let $X=\left(X_{1}, \ldots, X_{s+1}\right)$ and define

$$
S=\frac{1}{s}\left(X X^{T}-\bar{X} \bar{X}^{T}\right)
$$

as the sample covariance matrix, where $\bar{X}=\frac{1}{s+1} \sum_{j=1}^{s+1} X_{j}$ is the mean of the observations. For testing the hypothesis of sphericity John (1971) proposed to reject $H_{0}$ for large values of the statistic

$$
U=\frac{1}{n} \operatorname{tr}\left(\frac{S}{1 / n \operatorname{tr}(S)}-I_{n}\right)^{2}
$$

where $\operatorname{tr} A$ denotes the trace of the matrix $A$. For the problem of testing the hypothesis $H_{0}^{\prime}$ that the covariance matrix is given by the identity Nagao (1973) proposed to reject the hypothesis in (1.2) for large values of the statistic

$$
V=\frac{1}{n} \operatorname{tr}(S-I)^{2}
$$

Recently, Ledoit and Wolf (2002) investigated the properties of these tests if the sample size $s$ and the dimension $n$ converge to infinity at the same rate, that is

$$
\frac{n}{s} \rightarrow y \in(0, \infty)
$$

They proved asymptotic normality for an appropriately standardized version of $U$ and $V$ and showed that in this case the test of sphericity based on the statistic $U$ is still consistent, while the test for the hypothesis $H_{0}^{\prime}$, which rejects the null hypothesis for large values of the statistic $V$, is not necessarily consistent under assumption (1.6). For this reason Ledoit and Wolf (2002) proposed to reject the null hypothesis $H_{0}^{\prime}$ in (1.2) for large values of the statistic

$$
W=\frac{1}{n} \operatorname{tr}\left(S-I_{n}\right)^{2}-\frac{n}{s}\left(\frac{1}{n} \operatorname{tr}(S)\right)^{2}+\frac{n}{s}
$$

and showed asymptotic normality of a standardized version of $W$ and consistency of the corresponding test. The proof consists of an application of the delta-method to the vector $\left(\frac{1}{n} \operatorname{tr} S, \frac{1}{n} \operatorname{tr} S^{2}\right)$, for which the asymptotic normality has been established by Jonsson (1982).

In the present note we investigate the properties of the statistics $U, V$ and $W$ in the extreme cases where the sample size and dimension are not of the same order, that is

$$
\frac{n}{s} \rightarrow y \in\{0, \infty\} .
$$


We will demonstrate that in these cases appropriately standardized versions of the statistics $U, V$ and $W$ are still asymptotically normal distributed. However, the method used by Ledoit and Wolf (2002) to prove these results under assumption (1.6) is not applicable any more. These difficulties are illustrated in Section 2. In Section 3 we use a direct argument to establish asymptotic normality under the assumption (1.7), which is of its own interest and based on special structures of the Wishart matrix. This technique avoids the application of the delta-method and may be useful to investigate other properties of the sample covariance matrix. Finally, some of the more technical arguments are given in the Appendix [see Section 4].

\section{The proof based on the delta-method - revisited}

In this section we briefly explain the difficulties in the application of the delta-method to establish asymptotic normality of the statistics $U, V$ and $W$ under assumption (1.8). The variances of $\frac{1}{n} \operatorname{tr} S$ and $\frac{1}{n} \operatorname{tr} S^{2}$ can be calculated by standard methods, that is

$$
\begin{aligned}
\operatorname{Var}\left(\frac{1}{n} \operatorname{tr} S\right) & =\frac{2}{n s} \\
\operatorname{Var}\left(\frac{1}{n} \operatorname{tr} S^{2}\right) & =\frac{8}{n s}+\frac{20 n^{2}+20 n}{n^{2} s^{2}}+\frac{8 n^{3}+20 n^{2}+20 n}{n^{2} s^{3}} .
\end{aligned}
$$

Define

$$
F_{n}=\left(\begin{array}{c}
\frac{1}{n} \operatorname{tr}(S)-\mathrm{E}\left[\frac{1}{n} \operatorname{tr}(S)\right] \\
\frac{1}{n} \operatorname{tr}\left(S^{2}\right)-\mathrm{E}\left[\frac{1}{n} \operatorname{tr}\left(S^{2}\right)\right]
\end{array}\right),
$$

then the following result can either be established by similar methods as given in Jonsson (1982) or by the same arguments as given in the proofs of the results in Section 3.

Lemma 2.1. If assumption (1.8) is satisfied with $y=0$, then

$$
\sqrt{n s} F_{n} \stackrel{\mathcal{D}}{\longrightarrow} \mathcal{N}(0, A)
$$

where the asymptotic covariance matrix $A$ is defined by

$$
A=\left(\begin{array}{ll}
2 & 4 \\
4 & 8
\end{array}\right) \text {. }
$$

If assumption (1.8) is satisfied with $y=\infty$, the variances of both components of the vector $F_{n}$ are of different order.

It follows from Lemma 2.1 that the delta-method is not applicable in the case $y=\infty$, because we cannot standardize both components of $F_{n}$ simultaneously in order to obtain a nondegenerate limit distribution. Note that the first component $\operatorname{tr}\left(\frac{1}{n} S\right)$ requires a standardization with $\sqrt{n s}$ in order to obtain a positive limiting variance. However, such a standardization would yield for the variance of the second component

$$
\operatorname{Var}\left(\frac{\sqrt{n s}}{n} \operatorname{tr} S^{2}\right)=8 \frac{n^{2}}{s^{2}}+o\left(\frac{n^{2}}{s^{2}}\right)
$$


which converges to infinity. Moreover, even in the case $y=0$ the application of the delta-method with the asymptotic normality in Lemma 2.1 produces substantial difficulties. For this we consider examplarily the statistic $U$ which is obtained as

$$
U=g\left(\frac{1}{n} \operatorname{tr} S, \frac{1}{n} \operatorname{tr} S^{2}\right)
$$

where the function $g$ is defined by $g(x, y)=y / x^{2}-1$. Observing that

$$
E\left[\frac{1}{n} \operatorname{tr} S\right]=1, \quad E\left[\frac{1}{n} \operatorname{tr} S^{2}\right]=\frac{n+s+1}{s}
$$

[see Jonsson (1982)] we obtain that the random variable

$$
\sqrt{n s}\left(U-g\left(1, \frac{n+s+1}{s}\right)\right)
$$

is asymptotically normal distributed with mean 0 and variance

$$
\left(-2 \frac{n+s+1}{s}, 1\right)\left(\begin{array}{ll}
2 & 4 \\
4 & 8
\end{array}\right)\left(\begin{array}{c}
-2 \frac{n+s+1}{s} \\
1
\end{array}\right)=\left(\frac{n+1}{s}\right)^{2}=o(1) .
$$

Consequently, the standardization from the first part of Lemma 2.1 yields a degenerate limit distribution for the statistic

$$
\sqrt{n s}\left(U-\frac{n+1}{s}\right)
$$

in the case where $n / s \rightarrow 0$. Moreover, the same difficulties appear if this technique is used to derive the asymptotic distribution of the statistics $V$ and $W$. In the following section we use an alternative methodology to derive asymptotic properties of these statistics.

\section{The Wishart matrix}

Note that under the null hypothesis $H_{0}$ or $H_{0}^{\prime}$ the estimator $S$ in (1.3) has essentially (up to a scaling factor) an $n$-dimensional Wishart distribution with $s$ degrees of freedom. The following Lemma shows that $\operatorname{tr} S$ and $\operatorname{tr} S^{2}$ have rather simple representations in terms of $\chi^{2}$-distributed random variables. Throughout this paper the symbol $\stackrel{\mathcal{D}}{=}$ means equality in distribution.

Lemma 3.1. If $\mathcal{W}$ denotes an $n$-dimensional Wishart matrix with $s$ degrees of freedom and covariance matrix $\Sigma=I_{n}$, then

$$
\begin{gathered}
\operatorname{tr}(\mathcal{W}) \stackrel{\mathcal{D}}{=} \frac{1}{s} \sum_{i=1}^{\min \{n, s\}}\left(Y_{i}+X_{|s-n|+i}\right) \\
\operatorname{tr}\left(\mathcal{W}^{2}\right) \stackrel{\mathcal{D}}{=} \frac{1}{s^{2}} \sum_{i=1}^{\min \{n, s\}}\left(Y_{i}+X_{|s-n|+i}\right)^{2}+\frac{2}{s^{2}} \sum_{i=1}^{\min \{n, s\}-1} Y_{i} X_{|s-n|+i+1}
\end{gathered}
$$


where $X_{\ell} \sim \chi^{2}(\ell)$ and $Y_{i} \sim \chi^{2}(i)$ are independent chi-square distributed random variables.

As an application of Lemma 2.1 we can find very simple expressions for the statistics $U, V, W$ in terms of independent random variables with a $\chi^{2}$ distribution. These results can be used to obtain a direct proof of asymptotic normality based on a central limit theorem for $m$-dependent triangular arrays of random variables. We will illustrate the general strategy for the statistic $V$ in the case $n \geq s$, all other cases are treated similary. The following lemma gives an asymptotic equivalent representation for the statistic $V$. The proof is straightforward and therefore omitted.

Lemma 3.2. If the null hypothesis (1.2) is valid and $\lim _{n, s \rightarrow \infty} n / s=y \in[1, \infty]$ the statistic $V$ defined in (1.5) satisfies

$$
\sqrt{\frac{s^{3}}{n}}\left(V-\frac{n+1}{s}\right) \stackrel{\mathcal{D}}{=} \sum_{i=1}^{s} Y_{s, i}+A_{n} .
$$

Here $A_{n}=o_{p}(1)$ and the random variables $Y_{s, i}$ are given by

$$
Y_{s, i}=\left(\frac{1}{\sqrt{n^{3} s}}\left(V_{i}^{(n)}-\mathrm{E}\left[V_{i}^{(n)}\right]\right)-2 \sqrt{\frac{s}{n^{3}}}\left(Z_{i}^{(n)}-\mathrm{E}\left[Z_{i}^{(n)}\right]\right)\right)
$$

with $Z_{i}^{(n)}=Y_{i}+X_{n-s+i}, V_{i}^{(n)}=Z_{i}^{(n)^{2}}+2 Y_{i} X_{n-s+i+1}$, where $X_{i}, Y_{i}$ are defined in Lemma 3.1.

Theorem 3.3. If the null hypothesis (1.2) is valid and $\lim _{n, s \rightarrow \infty} n / s=y \in[1, \infty]$, then

$$
\sqrt{\frac{s^{3}}{n}}\left(V-\frac{n+1}{s}\right) \stackrel{\mathcal{D}}{\longrightarrow} \mathcal{N}\left(0, \frac{4}{y}+8\right),
$$

where $1 / \infty:=0$.

The following theorems contain the corresponding statement for the case

$$
\lim _{n, s \rightarrow \infty} n / s=y \in[0,1)
$$

and for the statistics $U$ and $W$ defined by (1.5) and (1.7), respectively. The proofs are similar and therefore omitted.

Theorem 3.4. If the null hypothesis (1.2) is valid and $\lim _{n, s \rightarrow \infty} n / s=y \in[0,1]$, then

$$
s\left(V-\frac{n+1}{s}\right) \stackrel{\mathcal{D}}{\longrightarrow} \mathcal{N}(0,4+8 y)
$$

Remark 3.5. It was proved by Nagao (1973) that the test, which rejects the hypothesis

$$
H_{0}^{\prime}: \Sigma=I_{n}
$$


for large values of the statistic $V$, is consistent if the dimension $n$ is fixed and the sample size $s$ converges to infinity. Ledoit and Wolf (2002) demonstrated that this statement is not correct if $n$ and $s$ converge at the same rate to infinity. Theorem 3.3 shows that the test is still consistent if

$$
\lim _{n, s \rightarrow \infty} n / s=0 .
$$

The following result shows that the test based on $W$ for the hypothesis $H_{0}^{\prime}$ and the test based on $U$ for the hypothesis of sphericity is always consistent independently of the value

$$
y=\lim _{n, s \rightarrow \infty} n / s \in[0, \infty]
$$

Theorem 3.6. If the null hypothesis (1.2) is valid and $\lim _{n, s \rightarrow \infty} n / s=y \in[0, \infty]$ then

$$
s\left(W-\frac{n s+s-2}{s^{2}}\right) \stackrel{\mathcal{D}}{\rightarrow} \mathcal{N}(0,4)
$$

Theorem 3.7. If the null hypothesis (1.1) of sphericity is valid and $\lim _{n, s \rightarrow \infty} n / s=y \in[0, \infty]$, then

$$
s\left(U-\frac{n+1}{s}\right) \stackrel{\mathcal{D}}{\longrightarrow} \mathcal{N}(0,4)
$$

Acknowledgements. The work of H. Dette was supported by the Deutsche Forschungsgemeinschaft (SFB 475, Komplexitätsreduktion in multivariaten Datenstrukturen). The authors would like to thank Isolde Gottschlich who typed numerous versions of the paper with considerable technical expertise.

\section{Appendix: Proofs}

Proof of Lemma 3.1. We will only consider the case $s \geq n$, the remaining case can be treated by similar arguments. By its definition an $n$-dimensional Wishart matrix with $s$ degrees of freedom and covariance matrix $\Sigma=I_{n}$ can be represented as

$$
\mathcal{W}=\frac{1}{s} Y Y^{T}
$$

where $Y=\left(Y_{i j}\right)_{i=1, \ldots, n}^{j=1, \ldots, s}$ is an $(n \times s)$ matrix with independent identically distributed entries, such that $Y_{i j} \sim \mathcal{N}(0,1)$. It now follows from Silverstein (1985) that the matrix $\mathcal{W}$ is orthogonally similar to a triangular matrix $\tilde{A}=\left(\tilde{a}_{i j}\right)_{i, j=1, \ldots, n}$ with entries

$$
\begin{aligned}
\tilde{a}_{i, i} & =\frac{1}{s}\left(\widetilde{Y}_{n-i+1}^{2}+\widetilde{X}_{s-i+1}^{2}\right), i=1, \ldots, n \\
\tilde{a}_{i, i+1} & =\frac{1}{s} \widetilde{Y}_{n-i} \widetilde{X}_{s-i+1}, \quad i=1, \ldots, n-1 \\
\tilde{a}_{i+1, i} & =\frac{1}{s} \widetilde{Y}_{n-i} \widetilde{X}_{s-i+1}, \quad i=1, \ldots, n-1,
\end{aligned}
$$


where $\tilde{Y}_{n}=0, \tilde{X}_{i}^{2} \sim \chi^{2}(i) ; \tilde{Y}_{i}^{2} \sim \chi^{2}(i)$ are independent $\chi^{2}$ distributed random variables $\left(\tilde{X}_{i} \geq\right.$ $\left.0 ; \tilde{Y}_{i} \geq 0\right)$. Therefore it is easy to see that the matrix $\mathcal{W}$ has the same eigenvalues as the triangular matrix $A=\left(a_{i j}\right)_{i, j=1, \ldots, n}$ with entries

$$
\begin{aligned}
a_{i, i} & =\tilde{a}_{n-i+1, n-i+1}=\frac{1}{s}\left(\widetilde{Y}_{i}^{2}+\widetilde{X}_{s-n+i}^{2}\right), \quad i=1, \ldots, n \\
a_{i, i+1} & =\tilde{a}_{n-i, n-i+1}=\frac{1}{s} \widetilde{Y}_{i} \widetilde{X}_{s-n+i+1}, \quad i=1, \ldots, n-1 \\
a_{i+1, i} & =\tilde{a}_{n-i+1, n-i}=\frac{1}{s} \widetilde{Y}_{i} \widetilde{X}_{s-n+i+1}, \quad i=1, \ldots, n-1
\end{aligned}
$$

Introducing the notation $X_{i}=\tilde{X}_{i}^{2}, Y_{i}=\tilde{Y}_{i}^{2}$ the assertion of the Lemma is now obvious.

Proof of Lemma 3.2. By Lemma 3.1 we have

$$
\begin{aligned}
\sqrt{\frac{s^{3}}{n}}\left(V-\frac{n+1}{s}\right) \stackrel{\mathcal{D}}{=} \sum_{i=1}^{s} & \left(\frac{1}{\sqrt{n^{3} s}}\left(V_{i}^{(n)}-\mathrm{E}\left[V_{i}^{(n)}\right]\right)\right. \\
& \left.-2 \sqrt{\frac{s}{n^{3}}}\left(Z_{i}^{(n)}-\mathrm{E}\left[Z_{i}^{(n)}\right]\right)\right) \\
& -\frac{1}{\sqrt{n^{3} s}}\left(Y_{s}^{2}-\mathrm{E}\left[Y_{s}^{2}\right]\right)-\frac{2}{\sqrt{n^{3} s}}\left(Y_{s} X_{n}-\mathrm{E}\left[Y_{s} X_{n}\right]\right) \\
& -\frac{2}{\sqrt{n^{3} s}}\left(Y_{s} X_{n+1}-\mathrm{E}\left[Y_{s} X_{n+1}\right]\right) \\
& +2 \sqrt{\frac{s}{n^{3}}}\left(Y_{s}-\mathrm{E}\left[Y_{s}\right]\right)
\end{aligned}
$$

and a straightforward but tedious calculation now shows that the last four terms are of order $o_{p}(1)$ [see Birke (2003)].

Proof of Theorem 3.3. By Lemma 3.2 we obtain the representation

$$
\sqrt{\frac{s^{3}}{n}}\left(V-\frac{n+1}{s}\right) \stackrel{\mathcal{D}}{=} \sum_{i=1}^{s} Y_{s, i}+A_{n}
$$

where $A_{n}=o_{p}(1)$ and the random variables $Y_{s, i}$ are defined in (3.1). Note that the random variables $\left\{Y_{s, i}\right\}_{i=1, \ldots, s}$ form a triangular array of rowwise $m$-dependent random variables. Moreover, $E\left[Y_{s, i}\right]=0$, and a straightforward but tedious calculation yields

$$
\lim _{n, s \rightarrow \infty} \operatorname{Var}\left(\sum_{i=1}^{s} Y_{s, i}\right)=\frac{4}{y}+8
$$

(see the remark at the end of this Appendix). A central limit theorem for $m$-dependent triangular arrays was given by Orey (1958), but we found the conditions of a central limit theorem for 
triangular arrays of $\alpha$-mixing random variables easier to check. To be precise we applied Theorem 2.1 of Liebscher (1996) to the sequence

$$
\left(\sum_{i=1}^{s} Y_{s, i}\right)_{s \in \mathbb{N}}
$$

For this we calculate

$$
\begin{aligned}
\mathrm{E}\left[Y_{s, i}^{4}\right]= & \frac{1}{s^{2} n^{6}} \mathrm{E}\left(V_{i}^{(n)}-\mathrm{E}\left[V_{i}^{(n)}\right]\right)^{4} \\
& -\frac{8}{s n^{6}} \mathrm{E}\left(V_{i}^{(n)}-\mathrm{E}\left[V_{i}^{(n)}\right]\right)^{3}\left(Z_{i}^{(n)}-\mathrm{E}\left[Z_{i}^{(n)}\right]\right) \\
& +24 \frac{1}{n^{6}} \mathrm{E}\left(V_{i}^{(n)}-\mathrm{E}\left[V_{i}^{(n)}\right]\right)^{2}\left(Z_{i}^{(n)}-\mathrm{E}\left[Z_{i}^{(n)}\right]\right)^{2} \\
& -\frac{32 s}{n^{6}} \mathrm{E}\left(V_{i}^{(n)}-\mathrm{E}\left[V_{i}^{(n)}\right]\right)\left(Z_{i}^{(n)}-\mathrm{E}\left[Z_{i}^{(n)}\right]\right)^{3} \\
& +\frac{16 s^{2}}{n^{6}} \mathrm{E}\left(Z_{i}^{(n)}-\mathrm{E}\left[Z_{i}^{(n)}\right]\right)^{4}
\end{aligned}
$$

and

$$
\begin{aligned}
\mathrm{E}\left[Y_{s, i}^{6}\right]= & \frac{1}{s^{3} n^{9}} \mathrm{E}\left(V_{i}^{(n)}-\mathrm{E}\left[V_{i}^{(n)}\right]\right)^{6} \\
& -\frac{12}{s^{2} n^{9}} \mathrm{E}\left(V_{i}^{(n)}-\mathrm{E}\left[V_{i}^{(n)}\right]\right)^{5}\left(Z_{i}^{(n)}-\mathrm{E}\left[Z_{i}^{(n)}\right]\right) \\
& +\frac{60}{s n^{9}} \mathrm{E}\left(V_{i}^{(n)}-\mathrm{E}\left[V_{i}^{(n)}\right]\right)^{4}\left(Z_{i}^{(n)}-\mathrm{E}\left[Z_{i}^{(n)}\right]\right)^{2} \\
& -\frac{160}{n^{9}} \mathrm{E}\left(V_{i}^{(n)}-\mathrm{E}\left[V_{i}^{(n)}\right]\right)^{3}\left(Z_{i}^{(n)}-\mathrm{E}\left[Z_{i}^{(n)}\right]\right)^{3} \\
& +\frac{240 s}{n^{9}} \mathrm{E}\left(V_{i}^{(n)}-\mathrm{E}\left[V_{i}^{(n)}\right]\right)^{2}\left(Z_{i}^{(n)}-\mathrm{E}\left[Z_{i}^{(n)}\right]\right)^{4} \\
& -\frac{192 s^{2}}{n^{9}} \mathrm{E}\left(V_{i}^{(n)}-\mathrm{E}\left[V_{i}^{(n)}\right]\right)\left(Z_{i}^{(n)}-\mathrm{E}\left[Z_{i}^{(n)}\right]\right)^{5} \\
& +\frac{64 s^{3}}{n^{9}} \mathrm{E}\left(Z_{i}^{(n)}-\mathrm{E}\left[Z_{i}^{(n)}\right]\right)^{6} .
\end{aligned}
$$

Because the distribution of the random variables $V_{i}^{(n)}$ and $Z_{i}^{(n)}$ is explicitly known we can evaluate $\sum_{i=1}^{s} E\left|Y_{s, i}\right|^{k} \quad(k=4,6)$ explicitly [see Birke (2003) for more details]. In the following we denote by $p_{j}(s, k)$ and $q_{j}(s, k)$ polynomials of degree $k \in \mathbb{N}$ and argument $s$, then we obtain by a tedious calculation

$$
\begin{aligned}
\sum_{i=1}^{s} \mathrm{E}\left[Y_{s, i}^{4}\right]= & \frac{p_{1}(s, 6)}{s n^{6}}+\frac{p_{2}(s, 5)}{s n^{5}}+\frac{p_{3}(s, 4)}{s n^{4}}+\frac{p_{4}(s, 3)}{s n^{3}} \\
& +\frac{p_{5}(s, 2)}{s n^{2}}+\frac{p_{6}(s, 1)}{s n}+\frac{p(s, 0)}{s}=O\left(\frac{1}{s}\right),
\end{aligned}
$$




$$
\begin{aligned}
\sum_{i=1}^{s} \mathrm{E}\left[Y_{s, i}^{6}\right]= & \frac{q_{1}(s, 9)}{s^{2} n^{9}}+\frac{q_{2}(s, 8)}{s^{2} n^{8}}+\frac{q_{3}(s, 7)}{s^{2} n^{7}}+\frac{q_{4}(s, 6)}{s^{2} n^{6}}+\frac{q_{5}(s, 5)}{s^{2} n^{5}} \\
& +\frac{q_{6}(s, 4)}{s^{2} n^{4}}+\frac{q_{7}(s, 3)}{s^{2} n^{3}}+\frac{q_{8}(s, 2)}{s^{2} n^{2}}+\frac{q_{9}(s, 1)}{s^{2} n}+\frac{q_{10}(s, 0)}{s^{2}} \\
= & o\left(\frac{1}{s}\right),
\end{aligned}
$$

Consequently it follows from Theorem 2.1 and equation (2.1) in Liebscher (1996)

$$
\sum_{i=1}^{s}\left(\frac{1}{\sqrt{n^{3} s}}\left(V_{i}^{(n)}-\mathrm{E}\left[V_{i}^{(n)}\right]\right)-2 \sqrt{\frac{s}{n^{3}}}\left(Z_{i}^{(n)}-\mathrm{E}\left[Z_{i}^{(n)}\right]\right)\right) \stackrel{\mathcal{D}}{\rightarrow} \mathcal{N}\left(0, \frac{4}{y}+8\right)
$$

which proves the assertion of Theorem 2.1.

\section{References}

T.W. Anderson (1984). An Introduction to Multivariate Statistical Analysis. Wiley, N.Y.

M. Birke (2003). Zufällige Matrizen bei multivariater Normalverteilung. Diplomarbeit, Fakultät für Mathematik, Ruhr-Universität Bochum (in German).

S. John (1971). Some optimal multivariate tests. Biometrika, 58: 123-127.

S. John (1972). The distribution of a statistic used for testing sphericity of normal distributions. Biometrika, 59: 169/173.

D. Jonsson (1982). Some limit theorems for the eigenvalues of a sample covariance matrix. J. Multivariate Anal., 12: 1-38.

O. Ledoit and M. Wolf (2002). Some hypothesis tests for the covariance matrix when the dimension is large compared to the sample size. Ann. Statist., 30 (4): 1081-1102.

E. Liebscher (1996). Central limit theorems for sums of $\alpha$-mixing random variables. Stochastics and Stochastic Reports, 59: 241-258.

R.J. Muirhead (1982). Aspects of Multivariate Statistical Theory. Wiley, New York.

H. Nagao (1973). On some test criteria for covariance matrix. Ann. Statist., 1: 700-709.

S. Orey (1958). A central limit theorem for $m$-dependent random variables. Duke Math. J., 25: 543-546 (1958).

P. Sebastini, E. Gussoni, I.S. Kohane, M.F. Ramoni (2003). Statistical challenges in functional genomics. Statistical Science 18, 33-70.

J.W. Silverstein (1985). The smallest eigenvalue of a large dimensional Wishart matrix. Ann. Probab., 13: 1364-1368. 